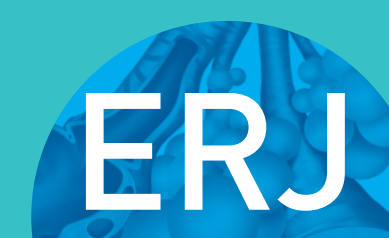

open research
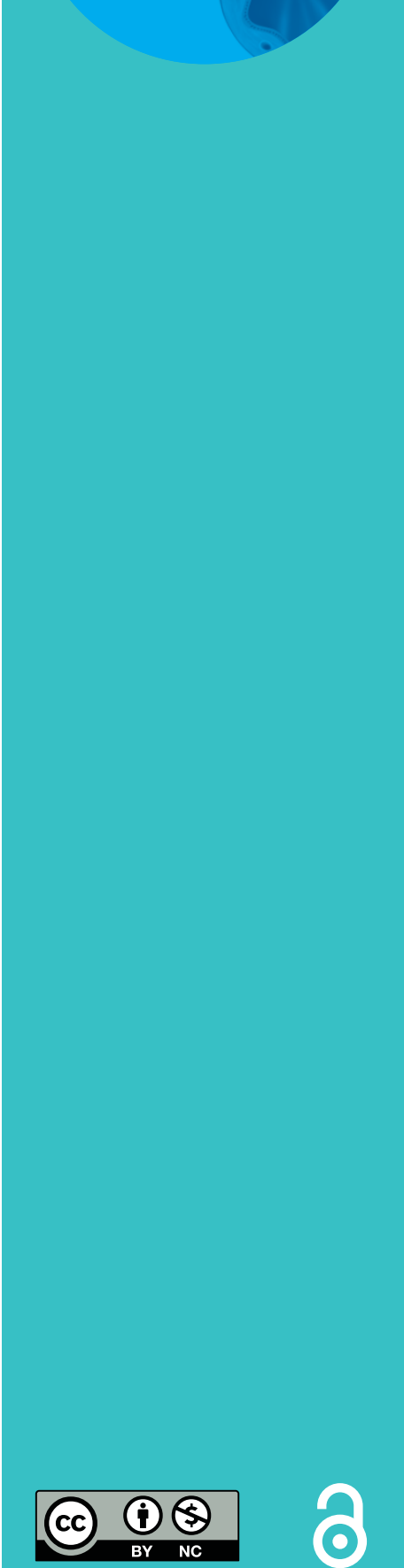

\section{Association of anti-tuberculosis drug concentrations in hair and treatment outcomes in MDR- and XDR-TB}

\author{
John Metcalfe ${ }^{1}$, Peter Bacchetti ${ }^{2}$, Roy Gerona ${ }^{3}$, Ali Esmail $^{4}$, Keertan Dheda $^{4}$ and \\ Monica Gandhi ${ }^{5}$
}

Affiliations: ${ }^{1}$ Division of Pulmonary and Critical Care Medicine, Zuckerberg San Francisco General Hospital and Trauma Center, University of California, San Francisco, CA, USA. ${ }^{2}$ Dept of Epidemiology and Biostatistics, University of California, UCSF, San Francisco, CA, USA. ${ }^{3}$ Maternal-Fetal Medicine Division, Dept of Obstetrics, Gynecology and Reproductive Sciences, University of California, UCSF, San Francisco, CA, USA. ${ }^{4}$ Lung Infection and Immunity Unit, Division of Pulmonology, University of Cape Town, Cape Town, South Africa. ${ }^{5}$ Division of HIV, Infectious Diseases and Global Medicine, Dept of Medicine, University of California, UCSF, San Francisco, CA, USA.

Correspondence: John Z. Metcalfe, Division of Pulmonary and Critical Care Medicine, Zuckerberg San Francisco General Hospital and Trauma Center, University of California, San Francisco, CA, USA. E-mail: john.metcalfeducsf.edu

ABSTRACT Therapeutic drug monitoring for drug-resistant tuberculosis (TB) is likely to improve treatment outcomes. While assessments of plasma drug levels can explain pharmacokinetic variability among trial participants, these measures require phlebotomy and a cold chain, and are generally not repeated frequently enough to characterise drug exposure over time. Using a novel multi-analyte assay, we found evidence that higher anti-TB drug concentrations in hair, a non-biohazardous and noninvasively collected biomatrix, predict extensively-drug resistant-TB clinical outcomes in a high-burden setting.

@ERSpublications

Anti-tuberculosis drug concentrations in hair, a non-biohazardous and noninvasively collected biomatrix, predict extensively drug-resistant-TB clinical outcomes in a high-burden setting. http://ow.ly/80xi30o8qSa

Cite this article as: Metcalfe J, Bacchetti P, Gerona R, et al. Association of anti-tuberculosis drug concentrations in hair and treatment outcomes in MDR- and XDR-TB. ERJ Open Res 2019; 5: 00046-2019 [https://doi.org/10.1183/23120541.00046-2019].

This article has supplementary material available from openres.ersjournals.com

Received: Feb 282019 | Accepted: March 052019

Copyright $\odot$ ERS 2019. This article is open access and distributed under the terms of the Creative Commons Attribution Non-Commercial Licence 4.0. 


\section{Introduction}

In 2017, the World Health Organization estimated that 558000 individuals developed multidrug-resistant tuberculosis (MDR-TB) [1]. Treatment outcomes remain poor, and result from clinical factors (HIV co-infection, disease severity), poor adherence to complicated regimens, low anti-TB drug concentrations from pharmacokinetic variability, suboptimal treatment regimens, or a combination of these. While assessments of plasma drug levels can explain pharmacokinetic variability among trial participants, these measures require phlebotomy and a cold chain, and are generally not repeated frequently enough to characterise drug exposure over time in real-world programmes. Measuring drug levels in dried blood spots circumvent some of these barriers, including the need for shipping samples via a cold chain, but assays for many MDR-TB drugs in dried blood spots have not been developed. A noninvasive method that does not require phlebotomy skills or biohazard precautions for long-term pharmacologic monitoring of multiple simultaneous MDR-TB drugs, particularly in patients with HIV infection, is likely to significantly advance the clinical management of MDR-TB.

Our group has extensive experience in the monitoring of antiretroviral concentrations in hair samples for patients with HIV, and have found that antiretroviral hair levels display linear relationships with dose within individuals [2], and are strong independent predictors of treatment outcomes [3]. In the field of TB, subtherapeutic concentrations of drugs are common (in particular among patients with MDR-TB [4-6] and those who are HIV co-infected [7-10]) and have been associated with acquired drug resistance [11-16], adverse treatment outcomes in drug-sensitive TB [17-19], increased target size (i.e. number of unique mutations conferring drug resistance) [20], and antagonistic (i.e. less than additive) drug interactions [21]. Furthermore, relative to antiretroviral combinations, TB regimens used for drug-resistant TB treatment are more toxic, less standardised, and may pose even greater challenges for adequate patient adherence [22].

\section{Methods}

We hypothesised that anti-TB drug concentrations in hair are associated with clinical outcomes among programmatically treated MDR-TB and extensively drug-resistant (XDR)-TB patients. Between July 1, 2016 and January 1, 2018, small, cosmetically unnoticeable hair samples (20-50 strands) were collected from 47 patients, the majority of whom were undergoing programmatic, inpatient, directly observed treatment for MDR- or XDR-TB at the Brooklyn Chest Hospital (Cape Town, South Africa). Of these, 46 (98\%) were female, 31 (66\%) were HIV-positive and 30 (64\%) had XDR-TB (with the remainder having MDR-TB). Patients were taking a median of five drugs (range 3-7), and the median amount of time on drugs prior to hair sampling was 87 days (interquartile range (IQR) 50-160 days). No participants were taking prothionamide, and only one participant (in a clinical trial) was taking pretomanid.

We analysed isoniazid (INH), ethambutol (EMB), pyrazinamide (PZA), levofloxacin (LFX), moxifloxacin (MFX), linezolid (LZD), clofazimine (CFZ), bedaquiline (BDQ), pretomanid and/or prothionamide in a multi-analyte panel using liquid chromatography/tandem mass spectrometry (LC-MS/MS) developed by our group [23]. Measured drug concentrations in hair are summarised in table 1. We calculated a Z-score for hair concentration of each drug (difference from the mean, divided by the standard deviation), and then averaged these for all drugs for a given participant to obtain their mean anti-TB drug Z-score. Because $3 \mathrm{~cm}$ of hair were assayed, representing $\sim 90$ days of hair growth, concentrations of drugs not

\section{TABLE 1 Observed anti-tuberculosis drug concentrations in small hair samples}

\begin{tabular}{lcccc} 
Drug & Patients $\mathbf{n}$ & Median $\mathbf{n g} \cdot \mathbf{m g}^{\mathbf{- 1}}$ & Quartile 1 & Quartile 3 \\
\hline Isoniazid & 22 & 1.67 & 0.30 & 2.47 \\
Pyrazinamide & 44 & 9.95 & 4.93 & 18.99 \\
Ethambutol & 34 & 1.15 & 0.69 & 2.01 \\
Levofloxacin & 28 & 35.16 & 23.76 & 59.99 \\
Moxifloxacin & 19 & 16.52 & 7.94 & 26.20 \\
Bedaquiline & 25 & 0.80 & 0.31 & 1.26 \\
Clofazimine & 31 & 0.82 & 0.51 & 1.45 \\
Linezolid & 22 & 11.22 & 3.89 & 41.71 \\
Ethionamide & 22 & 0.01 & 0.01 & 0.04 \\
Pretomanid & 1 & 1.14 & 1.14 & 1.14
\end{tabular}

Drug concentrations (in nanograms per milligram) are equal to those observed for individuals on the drug for the entire hair growth window (i.e. 3 months); levels were scaled up for those not on the drug for this duration. A refined adjustment was necessary for bedaquiline and clofazimine due to long half-life (refer to main text). 
taken for the entire 90 days before collection were scaled up by a factor of 90/Days, where Days was the number of days prior to collection that the drug was taken; only drugs that the patient was taking at the time of hair collection for at least 14 days were considered. For BDQ and CFZ, a more elaborate adjustment was used because of the long half-life of these drugs. From the first day of drug dosing, the contribution of each dose taken to the systemic plasma concentration during each day in the hair growth window was calculated, assuming rapid absorption of each dose followed by a half-life of 165 days for BDQ and 70 days for CFZ, and these were summed over all 90 days to obtain a total number of dose-days reflected in the hair sample (incorporation of drug into hair was assumed to be proportional to the systemic plasma concentration.) The measured concentrations were then multiplied by 1000/(total dose-days) to adjust for differing amounts of drug taken that should be reflected in the hair samples. Kaplan-Meier methods calculated the time to our a priori primary composite outcome of time from start of regimen to the first occurrence of treatment failure, relapse or death, and a Cox proportional hazards model assessed the effect of mean drug Z-score on this outcome. The levels of each measured drug were evaluated as individual predictors, restricted to those participants who were taking the drug at the time of hair collection and continued taking it until the outcome or the end of follow-up. Because INH, PZA, and CFZ concentrations were strongly right skewed, they were logarithmically transformed before Z-score calculation. Because of potential greater efficacy in the setting of MDR-TB, a secondary predictor combining only BDQ, LZD, MFX, LFX and CFZ (mean Z-score 5) was also assessed. A number of a priori defined two-predictor models were evaluated. Treatment outcome categories conformed to the definitions agreed for use by TB programmes for MDR-/XDR-TB, with the exception of loss to follow-up [24]. Loss to follow-up was a priori excluded from our composite outcome because of the uncertain relationship between sub-therapeutic drug exposure and treatment interruption, and the possibility that supra-therapeutic drug levels (and toxicity) could more plausibly account for an opposing association.

\section{Results}

An overall Kaplan-Meier curve is shown in figure 1. Median follow-up time after hair sampling was 354 days (IQR 43-482 days) among 23 individuals (out of the total sample size of 47) who were continuing treatment (13 were treated as right-censored observations) or who had been lost to follow-up (10 were also censored) by the end of the study. Median follow-up was 206 days (IQR 104-314 days) and 437 days (IQR 413-503 days) among those with a composite poor outcome (11 failure events) and those who were considered microbiologically cured (13 treated as never reaching the outcome), respectively. Univariate proportional hazards regression found suggestive evidence that higher drug concentrations in hair may be substantially associated with lower risk of poor treatment outcome (HR 0.60,95\% CI 0.27-1.34; $\mathrm{p}=0.21$ ), although this had wide uncertainty and did not reach statistical significance (table 2). Models that control for available covariates are shown in the supplementary material. Controlling for concomitant HIV led to a slightly larger effect size for hair concentration Z-score (HR 0.53), but others led to weaker effects (HR up to 0.71).

\section{Discussion}

We found preliminary evidence that anti-TB drug concentrations in hair, a novel and noninvasively collected biomatrix, are associated with clinical outcomes for drug-resistant TB among programmatically treated patients. Our panel assay, measuring 11 drugs used in second-line treatment regimens, was tested in scalp hair for individuals on multiple regimens at varying time-points during treatment. Our data

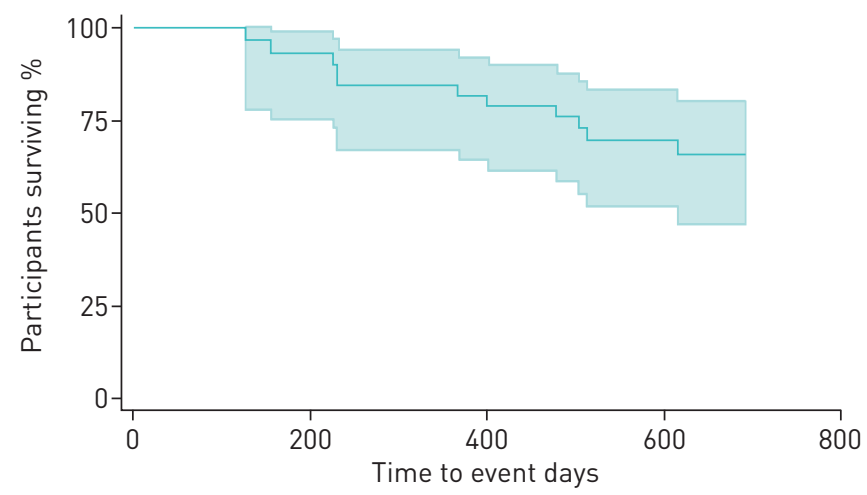

FIGURE 1 Time from hair sampling to composite outcome of treatment failure, relapse or death. KaplanMeier curves shows time to treatment failure, relapse or death among patients treated for multidrugresistant or extensively drug-resistant-tuberculosis at Brooklyn Chest Hospital (Cape Town, South Africa). Shaded area indicates the $\pm 95 \%$ confidence interval for the survival function. 


\begin{tabular}{|c|c|c|c|c|}
\hline Predictor & Patients n & Events $\mathrm{n}$ & $\operatorname{HR}(95 \% \mathrm{CI})$ & p-value \\
\hline \multicolumn{5}{|c|}{ Drug concentrations } \\
\hline Mean Z-score & 47 & 11 & $0.60(0.27-1.34)$ & 0.21 \\
\hline Isoniazid & 22 & 3 & $1.40(0.39-5.0)$ & 0.61 \\
\hline Pyrazinamide & 44 & 11 & $0.58(0.31-1.09)$ & 0.091 \\
\hline Ethambutol & 34 & 6 & $0.79(0.34-1.8)$ & 0.58 \\
\hline Levofloxacin & 28 & 5 & $1.21(0.52-2.8)$ & 0.66 \\
\hline Moxifloxacin & 19 & 6 & $0.95(0.42-2.2)$ & 0.91 \\
\hline Bedaquiline & 25 & 4 & $0.42(0.10-1.7)$ & 0.23 \\
\hline Clofazimine & 31 & 8 & $0.54(0.27-1.08)$ & 0.081 \\
\hline Linezolid & 22 & 3 & $0.55(0.10-3.1)$ & 0.50 \\
\hline \multicolumn{5}{|c|}{ Clinical and demographic risk factors } \\
\hline Age & 47 & 11 & $0.61(0.28-1.31)$ & 0.20 \\
\hline HIV-positive & 47 & 11 & $3.1(0.67-14.4)$ & 0.15 \\
\hline
\end{tabular}

suggest variability in hair concentrations for individual drugs likely due to individual pharmacokinetic variability (given that drugs were directly administered by nurses during inpatient treatment), and an effect size, despite not reaching statistical significance, suggesting a strong association between higher hair levels and better clinical outcomes.

Our results have strong biologic plausibility in that low anti-TB drug exposure predisposes to prolonged treatment, treatment failure, relapse and development of drug resistance [11, 25]. Although treatment in our study was directly administered to hospitalised patients, inter-individual pharmacokinetic variability is known to be influenced by body weight, sex, pharmacogenomics, comorbidities including HIV [26] and diabetes mellitus [27], and even drug manufacturer [28]. Despite significant inter-individual variability in drug exposure, as well clinical trials of TB therapeutics demonstrating the importance of pharmacodynamics [29], therapeutic drug monitoring of anti-TB drugs is not routinely employed, mainly due to feasibility issues. Hair collection is noninvasive and does not require phlebotomy skills, sterile equipment, a cold chain or biohazard precautions during collection or shipping, features which enhance feasibility in regions where resistant TB is prevalent.

A unique feature of hair levels relative to blood or urine concentrations is their ability to objectively assess drug exposure over prior weeks to months rather than hours to days [30]. Hair grows remarkably consistently in the occipital region of the scalp at approximately $1 \mathrm{~cm}$ per month, which allows hair to serve as a marker of time. The ability to measure average exposure to drugs, rather than assessing a snapshot of exposure via a shorter-term measure that may be influenced by intra-individual pharmacokinetics or adherence variability, is a major strength of therapeutic drug monitoring using hair levels. Moreover, hair can assess exposure over shorter periods of time than assessed in this paper (e.g. 1 month) and can be combined with plasma levels to provide information on patterns of drug exposure. Limitations, however, include that hair cannot be collected from individuals with shaved heads or very short hair, and that certain treatments, notably bleaching, can lower hair drug levels. Moreover, the assays described here require sophisticated equipment (LC-MS/MS), limiting current feasibility to research settings; however, lower cost assays using bench top systems are in development [31].

In conclusion, we provide promising evidence that noninvasive pharmacokinetic monitoring of small hair samples at pre-specified intervals may detect low anti-TB drug exposure in resistant TB infection, which could trigger interventions such as medication switches for drugs with very low levels or identifying drug-drug interactions prior to treatment failure, thereby prolonging regimen durability. As with conventional pharmacokinetic assessments, there is a need for randomised trials to assess the benefits of real-time hair level monitoring in the management of drug-resistant TB.

Conflict of interest: J. Metcalfe reports grants from NIH, during the conduct of the study. P. Bacchetti reports grants from NIH, during the conduct of the study. R. Gerona reports grants from NIH, during the conduct of the study. A. Esmail reports grants from NIH, during the conduct of the study. K. Dheda reports grants from NIH, during the conduct of the study. M. Gandhi reports grants from NIH, during the conduct of the study. 
Support statement: Funding for this work was provided by NIH/NIAID R01 AI123024 (principal investigators J. Metcalfe and M. Gandhi). Funding information for this article has been deposited with the Crossref Funder Registry.

\section{References}

1 World Health Organization. Global tuberculosis report 2018. Geneva, World Health Organization, 2018.

2 Liu AY, Yang Q, Huang Y, et al. Strong relationship between oral dose and tenofovir hair levels in a randomized trial: hair as a potential adherence measure for pre-exposure prophylaxis (PrEP). PLoS One 2014; 9: e83736.

3 Gandhi M, Ofokotun I, Bacchetti P, et al. Antiretroviral concentrations in hair strongly predict virologic response in a large HIV treatment-naive clinical trial. Clin Infect Dis 2019; 68: 1044-1047.

4 Mpagama SG, Ndusilo N, Stroup S, et al. Plasma drug activity in patients on treatment for multidrug-resistant tuberculosis. Antimicrob Agents Chemother 2014; 58: 782-788.

5 Barroso EC, Pinheiro VG, Facanha MC, et al. Serum concentrations of rifampin, isoniazid, and intestinal absorption, permeability in patients with multidrug resistant tuberculosis. Am J Trop Med Hyg 2009; 81: 322-329.

6 Heysell SK, Moore JL, Peloquin CA, et al. Outcomes and use of therapeutic drug monitoring in multidrug-resistant tuberculosis patients treated in Virginia, 2009-2014. Tuberc Respir Dis (Seoul) 2015; 78: 78-84.

7 McIlleron $\mathrm{H}$, Rustomjee $\mathrm{R}$, Vahedi $\mathrm{M}$, et al. Reduced antituberculosis drug concentrations in HIV-infected patients who are men or have low weight: implications for international dosing guidelines. Antimicrob Agents Chemother 2012; 56: 3232-3238.

8 Daskapan A, de Lange WC, Akkerman OW, et al. The role of therapeutic drug monitoring in individualised drug dosage and exposure measurement in tuberculosis and HIV co-infection. Eur Respir J 2015; 45: 569-571.

9 Gurumurthy P, Ramachandran G, Hemanth Kumar AK, et al. Decreased bioavailability of rifampin and other antituberculosis drugs in patients with advanced human immunodeficiency virus disease. Antimicrob Agents Chemother 2004; 48: 4473-4475.

10 Holland DP, Hamilton CD, Weintrob AC, et al. Therapeutic drug monitoring of antimycobacterial drugs in patients with both tuberculosis and advanced human immunodeficiency virus infection. Pharmacotherapy 2009; 29: 503-510.

11 Pasipanodya JG, McIlleron H, Burger A, et al. Serum drug concentrations predictive of pulmonary tuberculosis outcomes. J Infect Dis 2013; 208: 1464-1473.

12 Burman W, Benator D, Vernon A, et al. Acquired rifamycin resistance with twice-weekly treatment of HIV-related tuberculosis. Am J Respir Crit Care Med 2006; 173: 350-356.

13 Vernon A, Burman W, Benator D, et al. Acquired rifamycin monoresistance in patients with HIV-related tuberculosis treated with once-weekly rifapentine and isoniazid. Tuberculosis Trials Consortium. Lancet 1999; 353: $1843-1847$.

14 Benator D, Bhattacharya M, Bozeman L, et al. Rifapentine and isoniazid once a week versus rifampicin and isoniazid twice a week for treatment of drug-susceptible pulmonary tuberculosis in HIV-negative patients: a randomised clinical trial. Lancet 2002; 360: 528-534.

15 Weiner $\mathrm{M}$, Benator $\mathrm{D}$, Burman $\mathrm{W}$, et al. Association between acquired rifamycin resistance and the pharmacokinetics of rifabutin and isoniazid among patients with HIV and tuberculosis. Clin Infect Dis 2005, 40: 1481-1491.

16 Van Tongeren L, Nolan S, Cook VJ, et al. Therapeutic drug monitoring in the treatment of tuberculosis: a retrospective analysis. Int J Tuberc Lung Dis 2013; 17: 221-224.

17 Chideya S, Winston CA, Peloquin CA, et al. Isoniazid, rifampin, ethambutol, and pyrazinamide pharmacokinetics and treatment outcomes among a predominantly HIV-infected cohort of adults with tuberculosis from Botswana. Clin Infect Dis 2009; 48: 1685-1694.

18 Weiner M, Burman W, Vernon A, et al. Low isoniazid concentrations and outcome of tuberculosis treatment with once-weekly isoniazid and rifapentine. Am J Respir Crit Care Med 2003; 167: 1341-1347.

19 Prahl JB, Johansen IS, Cohen AS, et al. Clinical significance of $2 \mathrm{~h}$ plasma concentrations of first-line anti-tuberculosis drugs: a prospective observational study. J Antimicrob Chemother 2015; 70: 321-322.

20 Ford CB, Shah RR, Maeda MK, et al. Mycobacterium tuberculosis mutation rate estimates from different lineages predict substantial differences in the emergence of drug-resistant tuberculosis. Nat Genet 2013; 45: 784-790.

21 Ankomah P, Levin BR. Two-drug antimicrobial chemotherapy: a mathematical model and experiments with Mycobacterium marinum. PLoS Pathog 2012; 8: e1002487.

22 Daftary A, Padayatchi N, O’Donnell M. Preferential adherence to antiretroviral therapy over tuberculosis treatment: a qualitative study of drug-resistant TB/HIV co-infected patients in South Africa. Glob Public Health 2014; 9: 1107-1116.

23 Gerona R, Wen A, Koss C, et al. A multi-analyte panel for non-invasive pharmacokinetic monitoring of second-line anti-tuberculosis drugs. Int J Tuberc Lung Dis 2016; 20: 991-992.

24 World Health Organization. Definitions and reporting framework for tuberculosis - 2013 revision. Geneva, World Health Organization, 2014.

25 Sekaggya-Wiltshire C, von Braun A, Lamorde M, et al. Delayed sputum culture conversion in tuberculosis-human immunodeficiency virus-coinfected patients with low isoniazid and rifampicin concentrations. Clin Infect Dis 2018; 67: 708-716

26 Daskapan A, Idrus LR, Postma MJ, et al. A systematic review on the effect of HIV infection on the pharmacokinetics of first-line tuberculosis drugs. Clin Pharmacokinet 2018; [doi: 10.1007/s40262-018-0716-8].

27 Alkabab Y, Keller S, Dodge D, et al. Early interventions for diabetes related tuberculosis associate with hastened sputum microbiological clearance in Virginia, USA. BMC Infect Dis 2017; 17: 125.

28 Court R, Chirehwa MT, Wiesner L, et al. Quality assurance of rifampicin-containing fixed-drug combinations in South Africa: dosing implications. Int J Tuberc Lung Dis 2018; 22: 537-543.

29 McCallum AD, Sloan DJ. The importance of clinical pharmacokinetic-pharmacodynamic studies in unraveling the determinants of early and late tuberculosis outcomes. Int J Pharmacokinet 2017; 2: 195-212.

30 Baciu T, Borrull F, Aguilar C, et al. Recent trends in analytical methods and separation techniques for drugs of abuse in hair. Anal Chim Acta 2015; 856: 1-26.

31 Gandhi M, Yang Q, Bacchetti P, et al. Short communication: a low-cost method for analyzing nevirapine levels in hair as a marker of adherence in resource-limited settings. AIDS Res Hum Retroviruses 2014; 30: 25-28. 